Guilherme Assoni Gomes

(1) https://orcid.org/0000-0003-2998-0877

Luis Felipe Chaga Maronezi

(1) https://orcid.org/0000-0001-7947-939X

Giovana Bonessoni Felizari

Ohttps://orcid.org/0000-0002-1408-6285

Rogério Tomasi Riffel'

Ohttps://orcid.org/0000-0002-2285-204X

Jeanice de Freitas Fernandes 2

Ohttps://orcid. org/0000-0003-2137-7336

Renata dos Santos Rabello

Ohitps://orcid org/0000-0002-8966-4326

Ivana Loraine Lindemann

(O) https://orcid.org/0000-0002-6222-9746

\section{Caracterização dos óbitos por suicídio entre 2013-2017}

\author{
Characterization of deaths by suicide between 2013-2017
}

DOI: $10.1590 / 0047-2085000000335$

\section{RESUMO}

Objetivo: Descrever a frequência, a distribuição e as principais características dos casos de suicídio no Rio Grande do Sul. Métodos: Estudo transversal que incluiu todos os óbitos por suicídio notificados de 01/01/2013 a 31/12/2017. A análise compreendeu a descrição dos casos, a verificação da distribuição do método conforme o sexo e o cálculo das estimativas anuais de mortalidade. Resultados: Foram notificados 5.901 casos, a maioria do sexo masculino (79,3\%), com idade entre 35 e 59 anos (45,9\%), cor de pele branca $(90,9 \%)$, ensino fundamental $(66,6 \%)$, sem cônjuge $(63,6 \%)$ e trabalhadores do setor secundário (54,4\%). Do suicídio predominou ocorrência policial como fonte de notificação (90,8\%), domicílio como local de ocorrência (68,9\%), enforcamento, estrangulamento ou sufocação como causa básica (71\%); observaram-se diferenças significativas entre homens e mulheres quanto à escolha do método e aumento nas taxas anuais de mortalidade no período analisado. Conclusão: Constatou-se aumento dos óbitos por suicídio no local e período estudados, indicando que é uma importante causa de mortalidade, especialmente, entre homens adultos.

\section{PALAVRAS-CHAVE}

Suicídio, causas de morte, violência, notificação.

\section{ABSTRACT}

Objective: To describe the frequency, distribution and main characteristics of suicide cases in the state of Rio Grande do Sul. Methods: Cross-sectional study that included all deaths by suicide reported from 01/01/2013 to 12/31/2017. The analysis included the description of the cases, the verification of the distribution of the method according to sex and the calculation of annual mortality estimates. Results: 5,901 cases were reported, mostly male (79.3\%), aged between 35-59 years (45.9\%), white skin color (90.9\%), elementary school (66,6\%), without a spouse (63.6\%) and secondary sector workers (54.4\%). There was a predominance of police occurrences as a source of notification (90.8\%), home as the place of occurrence (68.9\%), hanging, strangulation or suffocation as a basic cause (71\%), significant differences were observed between men and women as to the choice of method and increase in annual mortality rates in the analyzed period. Conclusion: There was an increase in deaths by suicide in the studied place and period, indicating that it is an important cause of mortality, especially among adult men.

\section{KEYWORDS}

Suicide, causes of death, violence, notification.
Received in: Mar/03/2021. Approved in: Jun/07/2021

1 Federal University of Fronteira Sul, Passo Fundo Campus, Passo Fundo, RS, Brazil.

2 University of Passo Fundo, Passo Fundo, RS, Brazil. 


\section{INTRODUÇÃO}

O suicídio é caracterizado como um gesto autodestrutivo, com o intuito e o desejo de dar fim à própria vida. Também é descrito como um fenômeno de alta complexidade, com facetas e determinações múltiplas, que pode acometer todas as idades e classes sociais, de forma intencional e consciente, utilizando meios em que o suicida confia como sendo letais. É uma escolha ou ação que implica inúmeras e graves consequências e há vários motivos que podem levar um indivíduo a cometer suicídio; normalmente se observa a necessidade de aliviar pressões externas, tais como cobranças sociais, remorso, culpa, depressão e ansiedade, entre outras ${ }^{1,2}$.

Globalmente, cerca de 800 mil pessoas tiram suas vidas todos os anos refletindo em aproximadamente 2.150 suicídios diários. Conforme dados da Organização Mundial da Saúde (OMS), a taxa mundial de suicídio, padronizada por idade para 2016, foi de 10,5/100.000 habitantes, variando de 5 a mais de 30. No mesmo ano, o Brasil apresentou um índice de 6,1/100.000 habitantes e no Rio Grande do Sul ocorreram 1.166 casos, correspondendo a uma taxa de 11/100.000, sendo 17,8 em homens e 4,5 em mulheres, o que representa aproximadamente o dobro dos índices nacionais ${ }^{2-4}$.

Embora a situação seja preocupante, o suicídio pode ser prevenível. No Brasil, em 2015, o Centro de Valorização da vida (CW), o Conselho Federal de Medina (CFM) e a Associação Brasileira de Psiquiatria (ABP) iniciaram a campanha Setembro Amarelo, que é promovida todos os anos, em nível nacional, objetivando prevenir e reduzir os casos por meio do espaço junto à imprensa e da formação de parcerias com o intuito de aumentar a divulgação acerca da problemática e de conscientizar a população sobre a importância do tema. Ademais, o êxito na prevenção dos casos é favorecido com o incentivo à denúncia responsável, somado às políticas públicas relacionadas, em particular, à saúde mental. A precocidade da identificação dos possíveis casos e o manejo efetivo deles é a chave para que esses indivíduos recebam o cuidado do qual necessitam², ${ }^{2,6}$.

Diante disso, o objetivo deste trabalho foi descrever a frequência e a distribuição, assim como as principais características dos casos de suicídio em todos os municípios do Rio Grande do Sul (RS), pois, historicamente, o estado tem mostrado os mais elevados coeficientes de suicídio do país, e fatores como etnia, cultura, crises sociais e aspectos climáticos são levantados como possíveis fatores associados ao agravo?

\section{MÉTODOS}

Trata-se de um estudo transversal que incluiu todas as notificações de óbitos por suicídio de 01/01/2013 a 31/12/2017 no RS. A amostra foi selecionada a partir dos óbitos em que a variável 48 da Declaração de Óbito (DO) estava preenchida com a opção 2 (suicídio). Do banco de dados brutos do Sistema de Informações sobre Mortalidade (SIM), foram extraídas as seguintes variáveis: sexo, idade, cor da pele, situação conjugal, escolaridade, ocupação, fonte da notificação, causa básica do óbito, local e data de ocorrência e município de residência. Os dados em formato não agregado foram obtidos no Departamento de Gestão da Tecnologia da Informação - Núcleo de Informação em Saúde (NIS), em formato de planilha eletrônica, com supressão dos dados de identificação.

A análise compreendeu a descrição da amostra quanto a características dos casos e verificou-se a distribuição do método escolhido (causa básica) de acordo com o sexo das vítimas, por meio do teste do qui-quadrado, admitindo-se erro a de $5 \%$, sendo considerados significativos valores de $p$ $<0,05$, para testes bicaudais. Ainda, foram estimadas as taxas anuais de mortalidade por 100.000 habitantes no estado, as frequências relativas por Coordenadoria Regional de Saúde (CRS), bem como as taxas anuais de mortalidade por 100.000 habitantes por CRS, sendo os resultados apresentados de forma descritiva. Por fim, com o recurso de geoprocessamento, utilizando o software Terraview (versão 4.2.2, distribuição livre), foi possível mapear a ocorrência dos óbitos por suicídio nos 497 municípios do território estadual.

O estudo foi realizado em conformidade com a Resolução no 466/2012 do Conselho Nacional de Saúde, com aprovação do Comitê de Ética em Pesquisa com Seres Humanos da Universidade Federal da Fronteira Sul (Parecer no 3.189.541) e do Comitê de Ética da Escola de Saúde Pública, Secretaria Estadual de Saúde do Rio Grande do Sul (instituição coparticipante, sob o Parecer no 3.257.379).

\section{RESULTADOS}

No período analisado, foram notificados 5.901 óbitos por suicídio no estado e, conforme a Tabela 1, a maioria era do sexo masculino (79,3\%) e com idade entre 35 e 59 anos (45,9\%), sendo mais de $25 \%$ de idosos. Predominaram cor de pele branca (90,9\%), indivíduos sem cônjuge $(63,6 \%)$, com ensino fundamental incompleto/completo $(66,6 \%)$ e trabalhadores do setor secundário (54,4\%). No que tange às características relacionadas ao suicídio consumado, constatou-se que a principal fonte de notificação foi ocorrência policial $(90,8 \%)$ e que o principal local de ocorrência foi domiciliar (68,9\%). Em relação à causa básica do óbito, observou-se maior frequência de enforcamento, estrangulamento ou sufocação (71\%), seguida de morte por arma de fogo $(14,9 \%)$ e de intoxicação medicamentosa ou por outra substância (7\%).

Os números absolutos de casos e as taxas de mortalidade por 100.000 habitantes, por ano, estão apresentados na Figura 1. Observou-se uma curva ascendente, com aumento de, aproximadamente, 18\% entre os anos de 2013 e 2017. 
Tabela 1. Caracterização dos óbitos por suicídio ocorridos no estado do Rio Grande do Sul, de 2013 a $2017(n=5.901)$

\begin{tabular}{|c|c|c|}
\hline Variáveis & $\mathbf{n}$ & $\%$ \\
\hline \multicolumn{3}{|l|}{ Características das vítimas } \\
\hline \multicolumn{3}{|l|}{ Sexo } \\
\hline Masculino & 4.678 & 79,3 \\
\hline Feminino & 1.223 & 20,7 \\
\hline \multicolumn{3}{|l|}{ Idade (anos completos) } \\
\hline$\leq 17$ & 188 & 3,2 \\
\hline $18-34$ & 1.430 & 24,2 \\
\hline $35-59$ & 2.706 & 45,9 \\
\hline$\geq 60$ & 1.574 & 26,7 \\
\hline \multicolumn{3}{|l|}{ Cor da pele $(n=5.854)$} \\
\hline Branca & 5.323 & 90,9 \\
\hline Outras & 531 & 9,1 \\
\hline \multicolumn{3}{|l|}{ Situação conjugal ( $n=5.298$ ) } \\
\hline Sem cônjuge & 3.370 & 63,6 \\
\hline Com cônjuge & 1.928 & 36,4 \\
\hline \multicolumn{3}{|l|}{ Escolaridade $(n=3.526)$} \\
\hline Analfabetos & 146 & 4,1 \\
\hline Ensino fundamental & 2.350 & 66,6 \\
\hline Ensino médio ou mais & 1.030 & 29,2 \\
\hline \multicolumn{3}{|l|}{ Ocupação (n = 4.193) } \\
\hline Setor primário & 786 & 18,7 \\
\hline Setor secundário & 2.279 & 54,4 \\
\hline Setor terciário & 1.128 & 26,9 \\
\hline \multicolumn{3}{|l|}{ Características relacionadas ao suicídio } \\
\hline \multicolumn{3}{|l|}{ Fonte da notificação $(\mathrm{n}=4.956)$} \\
\hline Ocorrência policial & 4.497 & 90,8 \\
\hline Hospital & 159 & 3,2 \\
\hline Família & 66 & 1,3 \\
\hline Outra & 234 & 4,7 \\
\hline \multicolumn{3}{|l|}{ Causa básica do óbito } \\
\hline Enforcamento, estrangulamento ou sufocação & 4.187 & 71,0 \\
\hline Arma de fogo & 878 & 14,9 \\
\hline $\begin{array}{l}\text { Intoxicação medicamentosa ou por outra } \\
\text { substância }\end{array}$ & 415 & 7,0 \\
\hline Precipitação de local elevado & 139 & 2,4 \\
\hline Objeto cortante, penetrante ou contundente & 90 & 1,5 \\
\hline Afogamento & 85 & 1,4 \\
\hline Explosivos, fumaça, fogo ou chamas & 60 & 1,0 \\
\hline Impacto/permanência em veículo em movimento & 12 & 0,2 \\
\hline Outros meios não especificados & 35 & 0,6 \\
\hline \multicolumn{3}{|l|}{ Local de ocorrência ( $n=5.881)$} \\
\hline Domicílio & 4.050 & 68,9 \\
\hline Hospital & 670 & 11,4 \\
\hline Via pública & 376 & 6,4 \\
\hline Outros & 785 & 13,3 \\
\hline
\end{tabular}

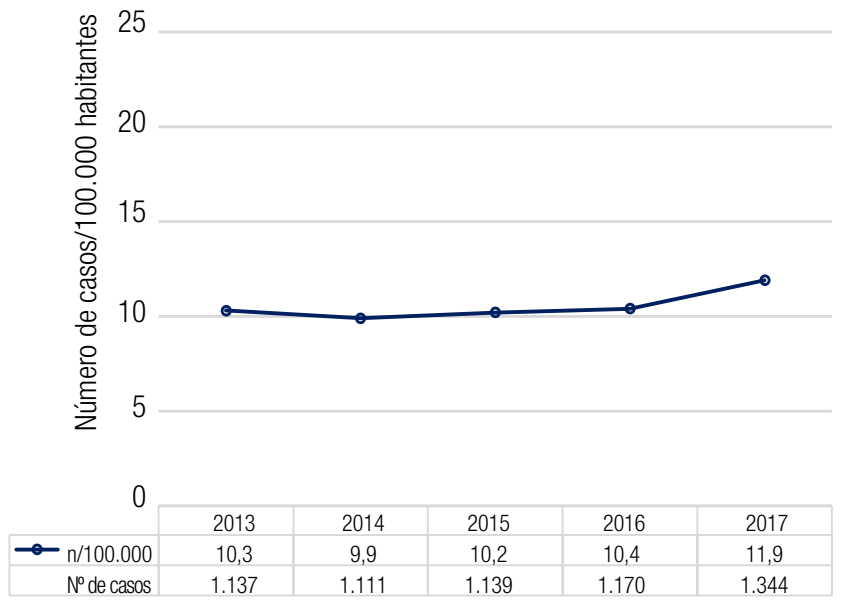

Figura 1. Suicídio no estado do Rio Grande do Sul de 2013 a 2017: números absolutos de casos e taxa de mortalidade/100.000 habitantes $(n=5.901)$.

Fonte: Sistema de Informações sobre Mortalidade.

A Tabela 2 apresenta as frequências absolutas e relativas de óbitos por suicídio ocorridos entre os anos de 2013 e 2017, em cada CRS do estado. Evidenciou-se maior frequência na $1^{\mathrm{a}}, 2^{\mathrm{a}}, 3^{\mathrm{a}}, 5^{\mathrm{a}}$ e $6^{\mathrm{a}} \mathrm{CRS}$, correspondendo às regiões de Porto Alegre (28,6\%), Pelotas (8,4\%), Caxias do Sul $(10,4 \%)$ e Passo Fundo (8,3\%), respectivamente. Ainda, estão demonstradas as taxas anuais de mortalidade/100.000 habitantes, em cada CRS, indicando valores elevados, em 2017, nas coordenadorias 13a , 19a $8^{a}$, 16a e 6a, correspondendo, respectivamente, a Santa Cruz do Sul, Frederico Westphalen, Cachoeira do Sul, Lajeado e Passo Fundo.

A Figura 2 representa a distribuição espacial do número de casos de suicídio de acordo com o município de ocorrência. Destacados com a cor preta aparecem os municípios que apresentaram elevado número de óbitos no período, como Canoas, Caxias do Sul, Novo Hamburgo, Passo Fundo, Pelotas, Porto Alegre, Rio Grande, Santa Cruz do Sul e Santa Maria.

A Tabela 3 apresenta a distribuição do método escolhido para o suicídio de acordo com o sexo das vítimas. Observou-se diferença estatisticamente significativa para: intoxicação medicamentosa; intoxicação por outras substâncias; enforcamento, estrangulamento ou sufocação; afogamento; arma de fogo; explosivos, fumaça, fogo ou chamas; e precipitação de local elevado. Desses, o método mais frequente em homens foi enforcamento, estrangulamento ou sufocação ( $p<$ $0,001)$ e uso de arma de fogo $(p=0,001)$. Já entre as mulheres houve maior prevalência de: intoxicação medicamentosa ( $p<0,001)$; intoxicação por outras substâncias $(p=0,043)$; afogamento ( $p=0,024)$; explosivos, fumaça, fogo ou chamas $(p<0,001)$; e precipitação de local elevado $(p=0,005)$.

Fonte: Sistema de Informações sobre Mortalidade. 
Tabela 2. Frequência de suicídios e taxas anuais de mortalidade por 100.000 habitantes por Coordenadoria Regional de Saúde (CRS): notificações no estado do Rio Grande do Sul, 2013-2017 $(n=5.901)$

\begin{tabular}{|c|c|c|c|c|c|c|c|}
\hline CRS & $\mathbf{n}$ & $\%$ & 2013 & 2014 & 2015 & 2016 & 2017 \\
\hline $1^{\mathrm{a}}-$ Porto Alegre & 711 & 12,0 & 8,04 & 6,81 & 8,45 & 9,63 & 6,87 \\
\hline $2^{a}-$ Porto Alegre & 980 & 16,6 & 8,39 & 5,33 & 5,92 & 6,52 & 9,46 \\
\hline $3^{\mathrm{a}}-$ Pelotas & 495 & 8,4 & 12,08 & 12,50 & 11,45 & 8,83 & 11,29 \\
\hline $4^{\mathrm{a}}-$ Santa Maria & 329 & 5,6 & 13,89 & 16,89 & 13,84 & 7,26 & 6,54 \\
\hline $5^{\mathrm{a}}$ - Caxias do Sul & 616 & 10,4 & 7,88 & 11,37 & 9,90 & 10,94 & 12,90 \\
\hline $6^{a}$ - Passo Fundo & 490 & 8,3 & 16,21 & 14,78 & 12,90 & 13,46 & 17,03 \\
\hline $7^{a}-$ Bagé & 92 & 1,6 & 8,98 & 11,07 & 8,42 & 8,93 & 11,02 \\
\hline $8^{a}$ - Cachoeira do Sul & 158 & 2,7 & 17,41 & 12,08 & 15,45 & 12,55 & 18,81 \\
\hline $9^{a}-$ Cruz Alta & 100 & 1,7 & 12,78 & 12,78 & 12,14 & 12,79 & 13,43 \\
\hline $10^{\mathrm{a}}$ - Alegrete & 227 & 3,8 & 8,59 & 7,34 & 11,76 & 8,83 & 11,15 \\
\hline $11^{\mathrm{a}}$ - Erechim & 160 & 2,7 & 12,60 & 12,59 & 11,75 & 13,84 & 16,35 \\
\hline $12^{\mathrm{a}}$ - Santo Ângelo & 178 & 3,0 & 8,18 & 9,90 & 20,53 & 13,02 & 9,26 \\
\hline $13^{\mathrm{a}}$ - Santa Cruz do Sul & 296 & 5,0 & 12,85 & 18,53 & 7,82 & 15,86 & 31,90 \\
\hline $14^{\mathrm{a}}$ - Santa Rosa & 180 & 3,1 & 11,16 & 17,19 & 15,05 & 18,08 & 15,94 \\
\hline $15^{\mathrm{a}}$ - Palmeira das Missões & 113 & 1,9 & 15,60 & 13,19 & 8,99 & 18,57 & 11,38 \\
\hline $16^{\mathrm{a}}$ - Lajeado & 265 & 4,5 & 15,79 & 13,96 & 14,17 & 14,10 & 18,61 \\
\hline $17^{\mathrm{a}}-$ ljuí & 119 & 2,0 & 7,36 & 12,54 & 10,36 & 11,64 & 9,47 \\
\hline 18a - Osório & 220 & 3,7 & 6,87 & 6,81 & 14,83 & 14,70 & 15,91 \\
\hline $19^{\mathrm{a}}$ - Frederico Westphalen & 160 & 2,8 & 13,57 & 13,59 & 13,61 & 23,07 & 19,95 \\
\hline Outras & 12 & 0,2 & - & - & - & - & - \\
\hline
\end{tabular}

Fonte: Sistema de Informações sobre Mortalidade.

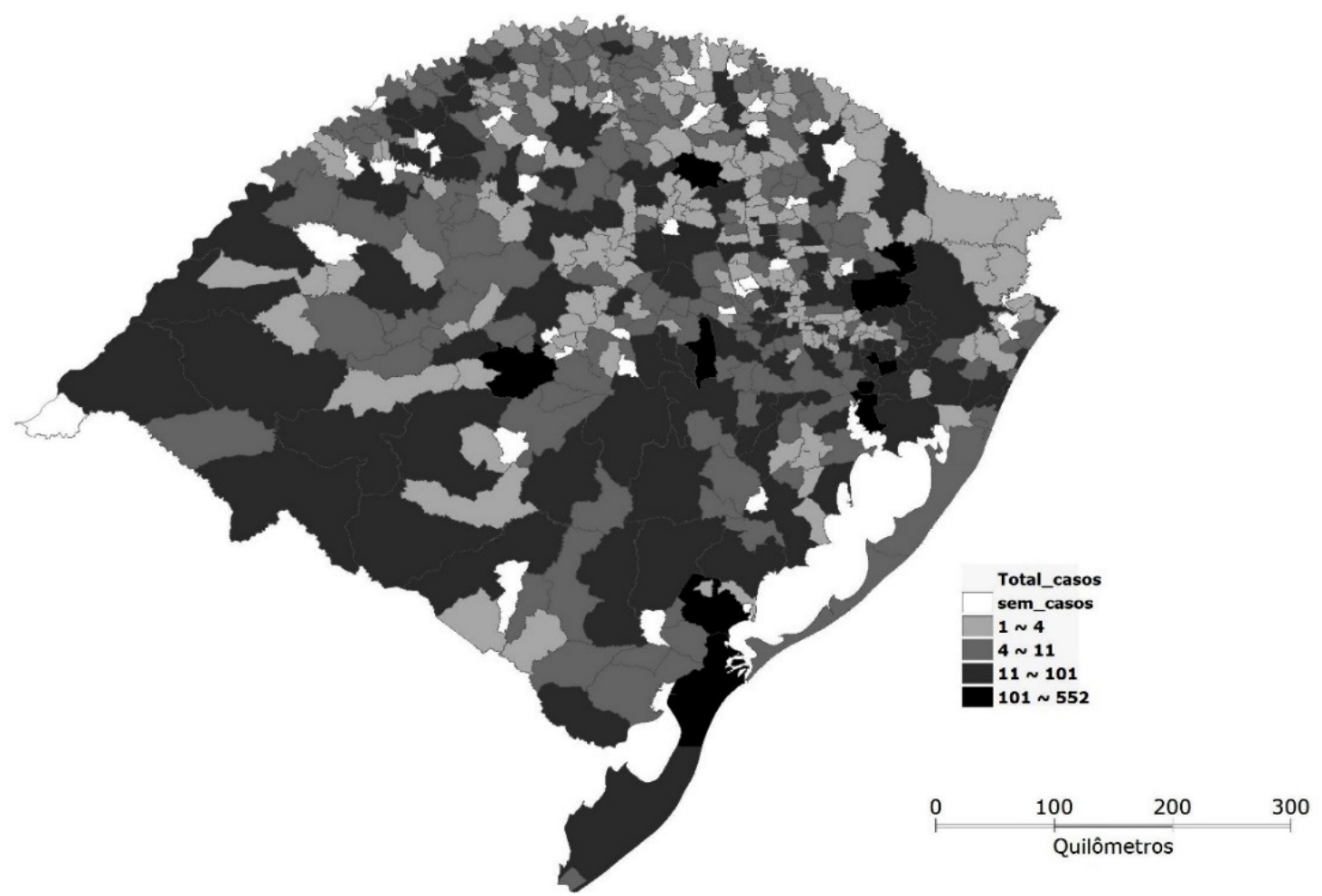

Figura 2. Óbitos por suicídio ocorridos no estado do Rio Grande do Sul de 2013 a 2017: distribuição por município de ocorrência $(n=5.901)$. Fonte: Sistema de Informações sobre Mortalidade. 
Tabela 3. Distribuição do método escolhido para o suicídio de acordo com o sexo das vítimas - Notificações no estado do Rio Grande do Sul, 2013-2017 $(\mathrm{n}=5.901)$

\begin{tabular}{|c|c|c|c|c|c|}
\hline \multirow{2}{*}{ Variáveis } & \multicolumn{2}{|c|}{ Masculino } & \multicolumn{2}{|c|}{ Feminino } & \multirow{2}{*}{$\mathbf{p}^{*}$} \\
\hline & n & $\%$ & $\mathbf{n}$ & $\%$ & \\
\hline Intoxicação medicamentosa & & & & & $<0,001$ \\
\hline Sim & 144 & 3,1 & 96 & 7,9 & \\
\hline Não & 4.534 & 96,9 & 1.127 & 92,1 & \\
\hline Intoxicações por outras substâncias & & & & & 0,043 \\
\hline Sim & 125 & 2,7 & 46 & 3,9 & \\
\hline Não & 4.553 & 97,3 & 1.177 & 96,1 & \\
\hline Enforcamento, estrangulamento ou sufocação & & & & & $<0,001$ \\
\hline Sim & 3.369 & 72,0 & 818 & 66,9 & \\
\hline Não & 1.309 & 28,0 & 405 & 33,1 & \\
\hline Afogamento & & & & & 0,024 \\
\hline Sim & 59 & 1,3 & 26 & 2,1 & \\
\hline Não & 4.619 & 98,7 & 1.197 & 97,9 & \\
\hline Arma de fogo & & & & & 0,001 \\
\hline Sim & 732 & 15,6 & 146 & 11,9 & \\
\hline Não & 3.946 & 84,4 & 1.077 & 88,1 & \\
\hline Explosivos, fumaça, fogo ou chamas & & & & & 0,001 \\
\hline Sim & 37 & 0,8 & 23 & 1,9 & \\
\hline Não & 4.641 & 99,2 & 1.200 & 98,1 & \\
\hline Objeto cortante, penetrante ou contundente & & & & & 0,223 \\
\hline Sim & 76 & 1,6 & 14 & 1,1 & \\
\hline Não & 4.602 & 98,4 & 1.209 & 98,9 & \\
\hline Precipitação de local elevado & & & & & 0,005 \\
\hline Sim & 97 & 2,1 & 42 & 3,4 & \\
\hline Não & 4.581 & 97,9 & 1.181 & 96,6 & \\
\hline Impacto/permanência em veículo em movimento & & & & & 0,289 \\
\hline Sim & 11 & 0,2 & 1 & 0,1 & \\
\hline Não & 4.667 & 99,8 & 1.222 & 99,9 & \\
\hline
\end{tabular}

* Teste do qui-quadrado.

Fonte: Sistema de Informações sobre Mortalidade.

\section{DISCUSSÃO}

Os resultados demonstram o crescimento do número de notificações no período, com predomínio em homens adultos, brancos, com baixa escolaridade, sem cônjuge e trabalhadores do setor secundário. Enforcamento, estrangulamento ou sufocação foram as causas mais frequentes, observando-se ainda maior ocorrência domiciliar e preferência por métodos mais violentos entre os homens. No que se refere ao incremento dos registros no período, o qual é demonstrado com a ascendência da curva (Figura 1), surge o questionamento acerca da explicação para o fato: aumento real do número de casos ou ampliação de notificações?

Diante disso, é preciso mencionar que, mesmo sendo obrigatório o preenchimento da causa da morte na DO, para os casos de suicídio, a notificação compulsória é recente, passando a ser realizada em 2011 de fato e, ampliada após a publicação da Portaria no 1.271/2014 do Ministério da Saúde ${ }^{8,9}$. Assim, é possível que o resultado seja decorrente de um conjunto de fatores, entre eles, a pertinência das políticas públicas, definidas por meio de portarias e de decretos, visando aprimorar o sistema de notificações ${ }^{10}$, como também os esforços mútuos de órgãos governamentais e não governamentais de saúde na realização de campanhas de prevenção ao suicídio, representando, dessa forma, o acréscimo de registros, e não, necessariamente, maior número de ocorrências.

Sobre os métodos escolhidos para o suicídio, pesquisa realizada no município de Sobral (CE) ${ }^{11}$ apontou maiores taxas de enforcamento e de uso de arma de fogo, consoante aos achados do presente estudo. Dados nacionais disponíveis no Boletim Epidemiológico do Ministério da Saúde ${ }^{12}$ 
indicam o enforcamento e a intoxicação exógena como causas mais frequentes. Cabe destacar, ainda, que os resultados ora apresentados demonstram que aproximadamente 70\% dos casos ocorreram no domicílio da vítima, o que pode estar relacionado tanto aos métodos escolhidos ${ }^{13}$ quanto à realização do ato de modo sigiloso, sem a possibilidade de intervenção. Nesse sentido, é recomendada como estratégia universal de prevenção ao suicídio a restrição de acesso aos principais meios, incluindo o controle de substâncias tóxicas e de armas de fogo ${ }^{14,15}$.

A maior frequência entre indivíduos do sexo masculino se deve ao fato de que essa parcela da população tende a alcançar a consumação do ato justamente por escolher meios mais violentos e letais, como, por exemplo, arma de fogo e enforcamento, ao passo que é recorrente entre as mulheres a intoxicação medicamentosa ou por outras substâncias ${ }^{4}, 0$ que corrobora a teoria da letalidade proposta por Mościcki em $1995^{16}$

No que tange à idade das vítimas, cerca da metade compreende gaúchos entre 35 e 59 anos (45,9\%), estando em concordância com dados nacionais disponibilizadas pelo Departamento de Informática do Sistema Único de Saúde (Datasus) $)^{17}$ para o ano de 2018 (53,8\% na mesma faixa etária). No entanto, ressalta-se que mais de $25 \%$ eram idosos, grupo naturalmente mais exposto a fatores de risco, os quais incluem morte de um ente querido - geralmente o cônjuge -, doença terminal, isolamento social, dependência física ou mental, violência financeira e psicológica, entre outros ${ }^{18}$.

Observou-se que a maioria das vítimas era de cor branca, sendo possível que o achado seja decorrente da tendência por parte dos profissionais de saúde, mesmo inconsciente, ao "embranquecimento" da vítima, visto que a cor da pele não é autodeclarada nesse caso. Ademais, o Boletim Epidemiológico do Ministério da Saúde ${ }^{12}$ traz à tona a questão das elevadas taxas de suicídio na população indígena, informação escassa no presente estudo, já que, conforme o último censo demográfico ${ }^{19}$, se considera a existência de 32.989 indígenas no Rio Grande do Sul.

O maior número de casos em indivíduos sem cônjuge $(63,6 \%)$ é previamente descrito na literatura, pois o vínculo afetivo, com menor propensão ao isolamento social, torna-se fator de proteção contra o suicídio ${ }^{20}$. Constatou-se, ainda, maior prevalência de suicidas analfabetos e com ensino fundamental incompleto/completo (70,7\%). A influência da baixa escolaridade é pouco abordada na literatura, porém alguns estudos destacam que, juntamente com a situação empregatícia e a renda familiar, é um dos critérios que define o "status econômico e social do indivíduo", causando distintos níveis de preocupações, estresse e frustração, colaborando para o sofrimento psíquico ${ }^{21-25}$.

Quanto às ocorrências por CRS no período, nota-se elevada frequência relativa naquelas sediadas nos municípios de Porto Alegre, Pelotas, Caxias do Sul e Passo Fundo, regionais correspondentes a grandes centros urbanos. Contudo, maiores taxas de mortalidade por 100.000 habitantes foram observadas nas regionais de Santa Cruz do Sul, Frederico Westphalen, Cachoeira do Sul e Lajeado e Passo Fundo. Em municípios de pequeno ou médio porte, os quais representam a maioria nessas últimas regionais, é possível que a dificuldade de implantação de programas de saúde mental e de medidas preventivas efetivas justifique os resul$\operatorname{tados}^{26}$. Ainda, é importante ressaltar que a população de tais municípios é composta por grande parcela de descendentes de europeus, além de que a economia é baseada na agricultura e, segundo evidências científicas, a exposição a altos níveis de pesticidas pode aumentar o risco de sequelas neuropsiquiátricas, incluindo transtornos de humor, depressão e ansiedade, assim como tentativas e suicídio consumado ${ }^{27,28}$.

Por fim, destaca-se que, por se tratar de estudo realizado com dados secundários oriundos das fichas de notificação, a principal limitação refere-se à qualidade deles, haja vista que a subnotificação, da mesma forma que o preenchimento incompleto ou inadequado dos instrumentos, pode acarretar uma descrição equivocada do agravo epidemiológico. Ainda assim, os resultados representam os registros oficiais do sistema de saúde e apontam para a magnitude do problema.

\section{CONCLUSÃO}

Apesar da possibilidade de que as taxas estejam subestimadas, os achados do presente estudo apontam elevados índices de suicídio no estado do Rio Grande do Sul, com destaque para algumas regiões e principalmente entre homens adultos e de cor branca.

Embora em muitos aspectos analisados os resultados corroborem os postulados da literatura, a descrição dos casos notificados, englobando tanto a caracterização das vítimas quanto à localização espacial das ocorrências, é fundamental para o direcionamento da atenção em saúde, servindo de subsídio para o planejamento, a implantação e o monitoramento de políticas públicas, com ênfase naquelas voltadas à saúde mental, de modo a prevenir as ocorrências, qualificar os atendimentos e melhorar a qualidade de vida da população, especialmente dos grupos de risco para o suicídio.

\section{CONTRIBUIÇÕES INDIVIDUAIS}

Guilherme Assoni Gomes - Contribuiu na concepção e desenho do estudo, na análise estatística, interpretação de dados, elaboração do artigo e aprovação da versão final a ser publicada.

Luis Felipe Chaga Maronezi - Contribuiu na concepção e desenho do estudo, revisão crítica do artigo e aprovação da versão final a ser publicada. 
Giovana Bonessoni Felizari - Contribuiu na concepção e desenho do estudo, revisão crítica do artigo e aprovação da versão final a ser publicada.

Rogério Tomasi Riffel - Contribuiu na análise estatística, interpretação de dados, revisão crítica do artigo e aprovação da versão final a ser publicada.

Jeanice de Freitas Fernandes - Contribuiu na concepção e desenho do estudo, revisão crítica do artigo e aprovação da versão final a ser publicada.

Renata dos Santos Rabello - Contribuiu na análise estatística, interpretação de dados, revisão crítica do artigo e aprovação da versão final a ser publicada.

Ivana Loraine Lindemann - Contribuiu na concepção e desenho do estudo, na análise estatística, interpretação de dados, revisão crítica do artigo e aprovação da versão final a ser publicada.

\section{CONFLITO DE INTERESSES}

Financiamentos: O primeiro autor declara ter recebido, durante 12 meses, bolsa financiada pela Fundação de Amparo à Pesquisa do Rio Grande do Sul (FAPERGS - Edital no 194/ GR/UFFS/2019), para participação no estudo.

O segundo autor declara ter recebido, durante 13 meses, bolsa financiada pelo Conselho Nacional de Desenvolvimento Científico e Tecnológico (CNPq - Edital no 335/GR/UFFS/2019), para participação no estudo.

O terceiro autor declara ter recebido, durante 12 meses, bolsa financiada pela Universidade Federal da Fronteira Sul (UFFS - Edital no 459/GR/UFFS/2019), para participação no estudo.

Todos os autores declaram não ter quaisquer conflitos de interesse com a realização do estudo.

\section{AGRADECIMENTOS}

Em especial, ao Centro Estadual de Vigilância em Saúde - Núcleo de Vigilância das Doenças e Agravos Não Transmissíveis, pelo suporte e disponibilização dos dados solicitados.

\section{REFERÊNCIAS}

1. Falando abertamente sobre suicídio [Internet]. Centro de Valorização da Vida; 2013 maio. Disponível em: https://crianca.mppr.mp.br/arquivos/File/publi/saúde/falando_ abertamente_sobre_suicidio.pdf. Acesso em: 13 set. 2020.

2. World Health Organization. Preventing suicide: A global imperative. [Internet]. 2014. Disponivel em: https://www.who.int/mental_health/suicide-prevention/world_ report_2014/em/. Acesso em: 13 set. 2020.
3. Conselho Nacional de Saúde (CNS). Um suicídio ocorre a cada 40 segundos no mundo [Internet]. 2019. Disponivel em: http://www.conselho.saude.gov.br/ultimas-noticiascns/809-um-suicidio-ocorre-a-cada-40-segundos-no-mundo-diz-organizacaomundial-da-saude. Acesso em: 13 set. 2020.

4. Centro Estadual de Vigilância em Saúde (CEVS). Boletim de Vigilância Epidemiológica de Suicídio e Tentativa de Suicídio [Internet]. 2018. Disponível em: https://www.cevs. rs.gov.br/upload/arquivos/201809/05162957-boletim-de-vigilancia-epidemiologica-desuicidio-n1-2018.pdf. Acesso em: 13 set. 2020.

5. Brasil. Ministério da Saúde. Prevenção do suicídio: sinais para saber e agir [Internet]. 2020. Disponível em: https://www.saude.gov.br/saude-de-a-z/suicidio. Acesso em: 13 set. 2020.

6. Associação Brasileira de Psiquiatria. Setembro Amarelo. 2020 Disponível em: https:// www.setembroamarelo.com/. Acesso em: 13 set. 2020.

7. Meneghel SN, Victora CG, Faria NMX, Carvalho LA, Falk JW. Características epidemiológicas do suicídio no Rio Grande do Sul. Rev Saúde Pública. 2004;38(6):804-10.

8. Brasil. Ministério da Saúde. Portaria no 1.271, de 6 de junho de 2014 [Internet]. Disponível em: http://bvsms.saude.gov.br/bvs/saudelegis/gm/2014/prt1271_06_06_2014.html. Acesso em: 13 set. 2020.

9. Palma DCA, Santos ES, Ignotti E. Análise dos padrões espaciais e caracterização dos suicídios no Brasil entre 1990 e 2015. Cad Saúde Pública. 2020;36(4):e00092819.

10. Cicogna JIC, Hillesheim D, Hallal ALLC. Mortalidade por suicídio de adolescentes no Brasil: Tendência temporal de crescimento entre 2000 e 2015. J Bras Psiquiatr. 2019;68(1):1-7.

11. Moreira RMM, Félix TA, Flôr SMC, Oliveira EN, Albuquerque JHM. Análise epidemiológica dos óbitos por suicídio. SANARE. 2017;16:29-34.

12. Secretaria de Vigilância em Saúde. Perfil epidemiológico das tentativas e óbitos por suicídio no Brasil e a rede de atenção à saúde. Boletim Epidemiológico [Internet]. 2017 [citado em 14 de set. de 2020];48 Disponível em: http://www.saude.gov.br/images/ pdf/2017/setembro/21/2017-025-Perfil-epidemiologico-das-tentativas-e-obitos-porsuicidio-no-Brasil-e-a-rede-de-atencao-a-saude.pdf. Acesso em: 13 set. 2020.

13. Souza PCZ, Souza AMRZ. Suicídio e trabalho: 0 que fazer? Cad Saúde Pública. 2010;26(12):2422-3.

14. World Health Organization. Public health action for the prevention of suicide: a framework [Internet]. Geneve: World Health Organization; 2012. 22p. Disponivel em: http://apps. who.int/iris/bitstream/10665/75166/1/9789241503570_eng.pdf?ud=1. Acesso em: 13 set. 2020.

15. Organização Panamericana de Saúde. Suicídio: Folha Informativa [Internet]. 2020 [citado em 18 de nov. de 2020]. Disponível em: https://www.paho.org/pt/topicos/suicidio. Acesso em: 13 set. 2020

16. Mościcki EK. Epidemiology of Suicidal Behavior. Suicide Life Threat Behav. 1995;25(1):2235.

17. Brasil. Ministério da Saúde. Banco de dados do Sistema Único de Saúde - Datasus, Informações de Saúde, Rede assistencial. Disponível em: http://www2.datasus.gov.br/ DATASUS/index.php. Acesso em: 13 set. 2020.

18. Minayo MCS, Cavalcante FG. Suicídio entre pessoas idosas: revisão da literatura. Rev Saúde Pública. 2010;44(4):750-7.

19. Censo Demográfico 2010. Características gerais da população, religião e pessoas com deficiência. Rio de Janeiro: IBGE; 2012. Disponível em: https://cens02010.ibge.gov.br/ resultados.html. Acesso em: 13 set. 2020.

20. Botega NJ. Comportamento suicida: epidemiologia. Psicol USP. 2014;25(3).

21. Mann M, Hosman CM, Schaalma HP, de Vries NK. Self-esteem in a broad-spectrum approach for mental health promotion. Health Educ Res. 2004;19(4):357-72.

22. Stevović LI, Jašović-Gašić M, Vuković 0 , Peković M, Terzić N. Gender differences in relation to suicides committed in the capital of Montenegro (Podgorica) in the period 2000-2006. Psychiatria Danubina. 2011;23(1):45-52.

23. Desaulniers J, Daigle MS. Inter-regional variations in men's attitudes, suicide rates and sociodemographics in Quebec (Canada). Soc Psychiatry Psychiatr Epidemiol. 2008;43(6):445-53.

24. Machado DB, Santos DN. Suicídio no Brasil, de 2000 a 2012. J Bras Psiquiatr. 2015;64:45-54. 
25. Gonçalves LRC, Gonçalves E, Oliveira-Júnior LB. Determinantes espaciais e socioeconômicos do suicídio no Brasil: uma abordagem regional. Nova Econ. 2011;21(2).

26. FranckMC, Monteiro MG, Limberger RP. Mortalidade por suicídio no Rio Grande do Sul: uma análise transversal dos casos de 2017 e 2018. Epidemiol Serv SaÚde. 2020;29(2):e2019512.
27. Faria NM, Fassa AG, Meucci RD. Association between pesticide exposure and suicide rates in Brazil. Neurotoxicology. 2014;45:355-62.

28. Freire C, Koifman S. Pesticides, depression and suicide: a systematic review of the epidemiological evidence. Int J Hyg Environ Health. 2013;216(4):445-60. 